\title{
Modelado y Simulación de la Degradación Fotocatalítica Heterogénea de 4-Clorofenol en un Reactor CPC a Escala Piloto
}

\author{
Miguel A. Mueses ${ }^{(1)}$, Fiderman Machuca-Martínez ${ }^{(2)}$ y Rubén J. Camargo-Amado ${ }^{(3)}$ \\ (1) Fotocatálisis e Ingeniería de Reactores Solares Fotocatalíticos, Departamento de Ingeniería Química, \\ Universidad de Cartagena, A.A. 1382 - Postal 195, Cartagena-Colombia \\ (e-mail: mmueses@unicartagena.edu.co) \\ (2) Grupo GAOX, Escuela de Ingeniería Química, Universidad del Valle, A.A. 25360, Cali-Colombia \\ (e-mail: fiderman.machuca@correounivalle.edu.co) \\ (3) Grupo de Fisicoquímica de Bio y Nanomateriales, Escuela de Ingeniería Química, Universidad del Valle, \\ A.A. 25360, Cali-Colombia (e-mail: ruben.camargo@correounivalle.edu.co)
}

Recibido Mar. 19, 2014; Aceptado May. 13, 2014; Versión final recibida May. 19, 2014

\section{Resumen}

Se presenta el modelado matemático y la simulación de la fotodegradación solar heterogénea del 4clorofenol (4-CP) en un reactor $\mathrm{CPC}$ a escala piloto utilizando $\mathrm{TiO}_{2}-\mathrm{P} 25$ como catalizador. El modelo matemático del proceso es un acople de modelos que describen la radiación solar incidente, la absorcióndispersión de fotones dentro del espacio de reacción, el balance de materia y una ecuación de velocidad de reacción generalizada para compuestos orgánicos, acoplada a un modelo predictivo de rendimientos cuánticos. Se incluyeron los efectos hidrodinámicos sobre el desempeño del reactor. Se realizaron comparaciones predictivas y se comparó con datos experimentales, obteniéndose un error global en la predicción inferior al $1.5 \%$. Estos resultados mostraron la viabilidad y extensión del enfoque matemático utilizado tanto para el reactor como para la cinética de degradación fotocatalítica del 4- clorofenol.

\section{Modelling and Simulation of the Heterogeneous Photocatalytic Degradation of 4-Chlorophenol in a Pilot-Scale CPC Reactor}

\begin{abstract}
Modeling and simulation of heterogeneous photocatalytic solar degradation of 4-chlorophenol (4-CP) in a CPC reactor at pilot-scale using $\mathrm{TiO}_{2}-\mathrm{P} 25$ as catalyst were presented. The mathematical model of the process is a coupling of incident solar radiation, scattering-absorption of the photons inside reactor, material balance and kinetic of generalized reaction rate and predictive quantum yields. The hydrodynamics effects on the reactor performance were included. Predictive values were compared with experimental data obtaining predictions with average error lower than $1.5 \%$. These results showed the feasibility and extend of the mathematical approach used for both the reactor and for the kinetics of catalytic degradation of 4chlorophenol.
\end{abstract}




\section{INTRODUCCIÓN}

La constante necesidad de remediar los efectos de la contaminación ambiental ha llevado a implementar tecnologías alternativas para el tratamiento de efluentes contaminantes provenientes de aguas residuales industriales. La fotocatálisis solar heterogénea es una tecnología avanzada de oxidación que ha sido aplicada para degradación de tintas, pesticidas, vinazas diluidas, compuestos clorados y contaminantes emergentes (Colina et al., 2009, Malato et al., 1999, 2000; Suaterna et al., 2012). El 4-clorofenol, contaminante presente en aguas residuales industriales de la producción de papel, plásticos, pesticidas e insecticidas ha sido objeto de estudio por aplicación de tecnologías avanzadas de oxidación, no obstante a escala laboratorio con lámparas UV artificiales, lo cual es una limitación fundamental para su viabilidad en aplicaciones a gran escala. En un trabajo previo (Suaterna et al., 2012) se evaluó la degradación del 4clorofenol a escala piloto solar alcanzándose hasta $70 \%$ de mineralización para energías acumuladas de $30 \mathrm{~W} / \mathrm{m}^{2}$.

El proceso de degradación del 4-clorofenol por fotocatálisis heterogénea ha sido modelado rigurosamente a escala laboratorio (Satuf et al., 2008) por análisis de los mecanismos de reacción y el acople del campo radiante, el cual es estimado por solución de la ecuación de transferencia radiativa (RTE) a través de la velocidad volumétrica local de absorción de fotones (LVRPA), responsable de la activación de las partículas de catalizador (Cassano y Alfano, 2000). Este enfoque es adecuado para la determinación de propiedades ópticas del semiconductor y de los parámetros cinéticos (Zalazar et al., 2005); sin embargo los tiempos computacionales son muy altos, debido a la complejidad matemática de las ecuaciones y no ha sido reportado su uso a escala solar.

Muy pocos reportes de modelos a gran escala para tratamiento de contaminantes orgánicos se encuentran en la literatura. Recientemente Mueses et al. (2013), propusieron un enfoque matemático para escala solar, soportado en el concepto de radiación efectiva, propiedades isotrópicas constantes (Machuca et al., 2008) y una ecuación de velocidad de reacción formulada a partir de un mecanismo de reacción basado en ataque de radicales hidroxilo y modificado con adsorción molecular. El modelo incluyó una variación de los rendimientos cuánticos en función de las condiciones de operación y el flujo luminoso del sistema. El modelo describió satisfactoriamente datos experimentales de mineralización solar de compuestos clorados, incluyendo el 4-clorofenol a 120 ppm de concentración inicial, $0.1 \mathrm{~g} / \mathrm{L}$ de catalizador en tres reactores CPC a escala piloto. En este trabajo extendie la aplicación del modelo propuesto por Mueses et al. (2013), en la fotodegradación a escala solar de 4-clorofenol en un reactor CPC, a diferentes condiciones de operación de $\mathrm{pH}$ y carga de catalizador para comprobar la efectividad del modelo reportado previamente.

\section{METODOLOGÍA}

Para el modelado matemático del reactor fotocatalítico heterogéneo solar a escala piloto se ha propuesto el siguiente esquema, basado en los cuatro componentes fundamentales de los procesos fotocatalíticos (Mueses y Machuca, 2010): i) campo radiante (fuente de radiación), ii) semiconductor, iii) Substrato de reacción y iv) Tipo de reactor. En la Figura 1 se muestra el esquema utilizado, la cual incluye la definición del sistema (I) que implica establecer: el tipo de catalizador, la fuente radiante, el substrato y el tipo de reactor; además de las condiciones de operación, como concentración inicial, carga de catalizador, flujo volumétrico, volumen de reactor (zona iluminada), volumen total de tratamiento, $\mathrm{pH}$ y oxígeno disuelto. La estructura metodológica implica una fase de modelado (II a III) y una fase de validación experimental (V). La fase modelado incluye el modelo matemático del reactor que acopla el modelo de radiación (emisión y absorción-scattering) a través del cálculo de la LVRPA, los balances de materia en zona de reacción y sistema de reciclo; el modelo de la velocidad de reacción y la hidrodinámica. Finalmente se acopla el modelo a una solución numérica (IV) para terminar con la etapa de simulación. Dado que en general los reactores fotocatalíticos son sistemas por lotes con reciclo, los efectos de la temperatura no son considerados; esta aproximación implica que no se requiere de un balance de energía térmica convencional.

\section{Modelo Matemático del Reactor}

El reactor fue modelado como un sistema de parámetros distribuidos en operación batch con reciclo. La radiación incidente sobre el eje axial fue considerada isotrópica pero anisotrópica sobre la dirección del eje $r$ dado que a escala solar la radiación incidente cuantificada experimentalmente es una variable global integrada en todas las longitudes de onda del espectro (Mueses et al, 2013). Lo anterior, admite una funcionalidad directa de la velocidad volumétrica de absorción de fotones (VRPA) en el plano de propagación (integral de área) más no una dependencia funcional directa con el volumen. Adicionalmente 
las siguientes consideraciones generales fueron hechas: i) Sistemas bifásicos con partículas sólidas de catalizador perfectamente esféricas $\left(\mathrm{TiO}_{2}\right.$ Degussa-P25), ii) Partículas de catalizador no poroso, iii) Sistemas de mezcla perfecta, distribución homogénea de componentes y partículas de catalizador en todo el volumen de reacción, iv) Flujos de fluido turbulentos, completamente desarrollados o perfectamente mezclados, $v$ ) Velocidad de reacción puntual, perfectamente homogénea en todo el volumen del reactor, vi) No hay presencia de gradiente de velocidad, vii) Solo existe absorción de energía por parte del catalizador, la fase fluida no absorbe radiación, viii) Campo de radiación en estado estable, ix) Espesor óptico despreciable a la entrada del sistema (paredes del reactor), $x$ ) Parámetros globales isotrópicos tanto de rendimiento cuántico como la velocidad volumétrica de absorción de fotones, OVRPA (Machuca et al, 2008; Mueses et al, 2013).

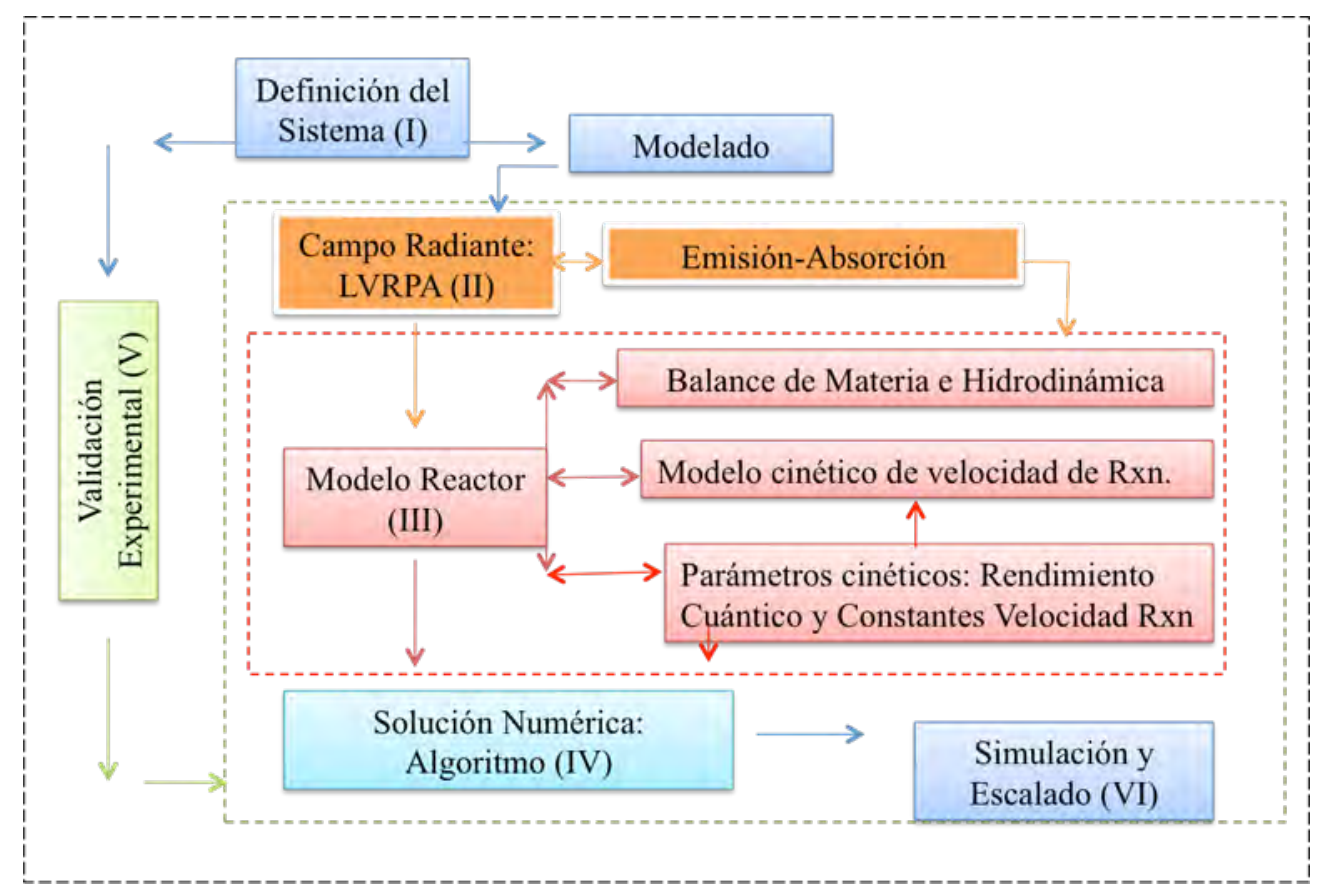

Fig. 1. Esquema para modelado y simulación de reactores solares heterogéneos a escala piloto.

\section{Balance de Materia}

La ecuación de balance de materia para el reactor solar en la zona de reacción (iluminada) es:

$$
\frac{\partial}{\partial t} C_{i}(t)=\left(\frac{v_{R}}{v_{T}}\right)\left\langle R_{i}\right\rangle_{R}(t)
$$

Donde $c_{i}$ es la concentración global en el tiempo $t$ del substrato $i,\left\langle R_{i}\right.$ es la velocidad promedio de reacción y $U_{R}, U_{T}$, son los volúmenes de reacción (expuesto a la radiación) y total del sistema (reactor más reciclo). Puesto que la radiación solar incidente no es constante con el tiempo por efecto de variaciones atmosféricas, localización geográfica y tiempo del año, se utilizó una estandarización del tiempo de tratamiento $t_{30 \mathrm{w}}$, para una intensidad de radiación de $30 \mathrm{~W} / \mathrm{m}^{2}$ estándar promedio en un día claro y soleado (Colina-Marquez et al, 2010, 2009; Mueses et al, 2013):

$$
t_{30 W, i+1}=t_{w 0 W, i}+\left(t_{i+1}-t_{i}\right) \frac{Q_{U V}}{30}\left(\frac{v_{R}}{v_{T}}\right)
$$

Quv es la intensidad de radiación solar promedio $\left(\mathrm{W} / \mathrm{m}^{2}\right)$ durante el intervalo de tiempo de toma de muestra. El balance de materia puede expresarse como una ecuación por paso de reacción en estado estable evaluado para cada tiempo espacial $T_{\text {pass }}$ (tiempo de residencia), en función de la longitud axial $z$. Esta aproximación describe el comportamiento dinámico del reactor batch como una suma finita de pasos ( $N_{\text {Pass,Total }}$ ) por el reactor, en el tiempo total de tratamiento para una energía total acumulada $Q_{U V \text {,Total }}$ (Mueses et al, 2013): 


$$
N_{\text {Pass, Total }}=\frac{t_{\text {Total,QUVacum,Total }}}{\tau_{\text {pass }}}
$$

Utilizando la ecuación de continuidad para un volumen diferencia $\delta u$ y el cálculo elemental; la ecuación de balance de materia para el sistema aplicada para cada paso por el reactor, en función de la posición espacial en $z$, se transforma fácilmente:

$$
\left.\frac{\partial c_{i}}{\partial Z}\right|_{\tau \text { pass }}=\frac{1}{v_{z, a v}}\left(\frac{1}{v_{T}}\right) \int_{v_{R}} r_{i}(\chi) d v=\frac{1}{v_{z}}\left(\frac{v_{R}}{v_{T}}\right)\left\langle R_{i}\right\rangle_{z}
$$

Donde $z$ es la coordenada axial y $v_{z, a v}$ es la velocidad promedio global en la dirección del mismo eje, $x$ es el vector posición del punto de reacción con coordenadas $(r, \theta, z) ;\langle R i\rangle_{z}$ la velocidad promedio para un punto espacial sobre el eje axial. Se requiere entonces acoplar la concentración de salida del reactor para cada paso $T_{\text {pass }}$ con la concentración de entrada que sale del sistema de tuberías y tanque de almacenamiento de la zona de reciclo. Un balance de materia simple en la zona oscura genera la siguiente relación para la concentración de entrada para cada paso por el reactor (Mueses et al, 2013):

$$
c_{i, \tau \text { pass }+1}=\frac{c_{i}\left(t-\tau_{\text {pass }}\right)\left[v_{T}-v_{R}\right]+c_{i}\left(\tau_{\text {pass }}\right) v_{R}}{v_{T}}
$$

\section{Ecuación de la Velocidad de Reacción}

Un modelo de velocidad de reacción propuesto por Mueses y colaboradores (Mueses et al, 2013) fue utilizada para la simulación de la degradación fotocatalítica heterogénea del 4-cloro fenol con $\mathrm{TiO}_{2}-\mathrm{P} 25$ suspendido. Esta ecuación es un modelo generalizado que ha sido aplicado en sistemas orgánicos simples, mezclas multicomponentes y pesticidas, con excelentes aproximaciones a los datos experimentales (Mueses et al, 2013).

$r_{i}=-2 \frac{\alpha_{1}}{\kappa_{P}}\left[-1+\sqrt{1+\frac{\kappa_{P}}{\alpha_{1}} \Phi_{g} \hat{E}_{g\left(\lambda \rightarrow \lambda^{\prime}\right)}^{a}}\right] \theta_{i}^{L-H}$

Con

$$
\begin{aligned}
& \theta_{i}^{L-H}=\frac{k_{A}^{L-H} C_{i}}{1+k_{A}^{L-H} C_{i}} \\
& \kappa_{P}=\frac{2}{S_{g} C_{C a t}}
\end{aligned}
$$

Con $r_{i}$ la velocidad de reacción $\left(\mathrm{mol} / \mathrm{m}^{3} \cdot \mathrm{s}\right), S_{g}$ el área superficial por masa de catalizador $\left(\mathrm{m}^{2} / \mathrm{kg}\right), C_{C a t}$ la carga de catalizador $\left(\mathrm{kg} / \mathrm{m}^{3}\right), c_{i}$ la concentración del substrato en $\left(\mathrm{mol} / \mathrm{m}^{3}\right), \kappa_{P}$ la constante de partícula $\left(\mathrm{m}^{3} / \mathrm{m}^{2}\right), \Phi_{g}$ el rendimiento cuántico efectivo global (mol/Einstein), $\hat{E}_{g\left(\lambda \rightarrow \lambda^{\prime}\right)}^{a}$ la velocidad volumétrica global de absorción de fotones, OVRPA (Einstein $/ \mathrm{m}^{3} \cdot \mathrm{s}$ ) (Machuca et al, 2008), $k^{L-H}{ }_{A}$ una constante cinética tipo Langmuir-Hinshelwood $\left(\mathrm{m}^{3} / \mathrm{mol}\right)$ y $\alpha_{1}$ la constante cinética de velocidad de reacción $\left(\mathrm{mol} / \mathrm{m}^{2} \cdot \mathrm{s}\right)$. La ecuación cinética posee dos parámetros ajustables $\alpha_{1}$ y $k^{L-H}$. El rendimiento cuántico del sistema fue estimado utilizando el modelo de rendimiento cuántico efectivo propuesto por Mueses y colaboradores en 2013:

$\Phi_{g}=\Phi_{g}^{E f f} \cdot \Psi$

\section{Modelo Hidrodinámico}

El modelo hidrodinámico del reactor CPC solar se consideró para fluido turbulento (Re>65000) completamente desarrollado sin efectos viscosos. Las ecuaciones hidrodinámicas fueron tomadas de la literatura (Mueses et al, 2013): 


$$
\begin{aligned}
& \frac{v_{z}(r, \theta)}{v_{\max }}=\left(1-\frac{r}{R}\right)^{1 / \eta_{z}} \\
& \eta_{z}=0.41 \sqrt{\frac{8}{f}} \\
& f=\frac{0.0076 \mathrm{Re}^{\star 0.165}}{1+\mathrm{Re}^{\star 7.0}}+\frac{16}{\mathrm{Re}} \\
& \mathrm{Re}^{*}=3170 / \mathrm{Re}
\end{aligned}
$$

\section{Modelo Matemático del Campo Radiante}

Emisión: La radiación incidente solar sobre el reactor $I_{0}$ se calculó utilizando la metodología propuesta por Colina Márquez y Colaboradores $(2010,2009)$. Se consideró radiación solar constante en la dirección axial $z$ de los reactores (con radiación UV directa entre 295-384 nm y un 4.1\% del total de la radiación solar); con intensidad total de radiación $I_{0 \text {, Total }}$ fija en $30 \mathrm{~W} / \mathrm{m}^{2}$; la relación entre radiación difusa a directa se consideró constante y se fijó un $75 \%$ de transmitancia UV a través de las nubes. La radiación directa y difusa se corrigió por la posición geográfica y tiempo del año, así:

$\frac{I_{0, \text { direct }}}{I_{0, \text { Total }}}=1-\frac{I_{0, \text { diff }}}{I_{0, \text { Total }}}=1-\frac{4}{3} K_{C} \cdot h_{d}$

Siendo $K_{C}$ la relación de nubosidad evaluada con respecto a la radiación $H$ recibida en la localización de interés $\left(K c=H / H_{0}\right)$ y $h_{d}$ la corrección de energía difusa a total:

$$
\begin{aligned}
h_{d}= & 1-\frac{H_{d}}{H_{0}}= \begin{cases}0.01 & \text { para } K_{c} \leq 0.17 \\
-0.188+2.272 K_{c}-9.473 K_{c}^{2}+21.856 K_{c}^{3}-14.648 K_{C}^{4} \quad 0.17<K_{C} \leq 0.80\end{cases} \\
H_{0}= & 24 \frac{S}{\pi} E_{0}\left[h_{\mathrm{a}}-\tan \left(h_{\mathrm{a}}\right)\right] \sin (\varphi) \sin (\varpi) \\
E_{0}= & 1+0.033 \cos \eta_{d y} \\
\varpi= & 0.006918-0.399912 \cos \left(\eta_{d y}\right)+0.070257 \sin \left(\eta_{d y}\right)-0.006758 \cos \left(2 \eta_{d y}\right)+ \\
& 0.000907 \sin \left(2 \eta_{d y}\right)-0.002697 \cos \left(3 \eta_{d y}\right)+0.00148 \sin \left(3 \eta_{d y}\right) \\
\eta_{t y}= & \frac{2 \pi d_{y}}{365} \\
h_{\mathrm{a}}= & \arccos (-\tan \varphi \tan \varpi)
\end{aligned}
$$

Donde $\phi$ es la latitud geográfica, $\eta_{t y}$ es la fracción del año para el día $d_{y}$ expresada como ángulo (ejm. $d_{y}=1$ para 01 de enero), $\tilde{\omega}$ es el ángulo de declinación y $h_{\mathrm{a}}$ la hora solar angular. $H_{0}$ es la radiación solar global calculada para un día claro soleado, $E_{0}$ un factor de corrección de excentricidad y $S$ la constante solar $(S=$ $1.367 \mathrm{KWh} / \mathrm{m}^{2}$ ).

Modelo de Absorción-Dispersión de Radiación: El cálculo de la adsorción-scattering de radiación evaluada a través de la velocidad volumétrica de adsorción de fotones (LVRPA), calculada utilizando el modelo SFM (Six-Flux Scattering-Absorption Model) (Mueses et al, 2013; Colina-Marquez, et al, 2010 y 2009, Li Puma y Brucato, 2007):

$$
L V R P A=\frac{I_{0}}{\lambda_{\text {Corr }} \omega_{\text {Corr }}(1-\Gamma)}\left[\left(\omega_{\text {Eff }}-1+\sqrt{1-\omega_{\text {Corr }}^{2}}\right) e^{\frac{-r_{P}}{\lambda_{\text {Corr }}}}+\Gamma\left(\omega_{\text {Corr }}-1-\sqrt{1-\omega_{\text {Corr }}^{2}}\right) e^{\frac{r_{P}}{\lambda_{\text {Corr }}}}\right]
$$


Donde $I_{0}$ es una energía de radiación incidente sobre la pared del reactor; $r_{p}^{\prime}$ es la coordenada puntual en el espacio de reacción y $\omega_{\text {corr }}, \lambda_{\text {corr }}$, son el coeficiente de dispersión de albedo y la longitud de extinción, $\mathrm{k}$ el coeficiente volumétrico de absorción global $\left(\mathrm{m}^{-1}\right)$ y $\sigma$ el coeficiente volumétrico de scattering global $\left(\mathrm{m}^{-1}\right)$. Los parámetros ópticos para la LVRPA se calcularon de manera análoga al trabajo de Colina-Márquez teniendo en cuenta las propiedades del catalizador $\mathrm{TiO}_{2}-\mathrm{P} 25$ (Degussa-Evonik) evaluadas a las condiciones de radiación solar incidente para la Ciudad de Cali, Colombia, en el año 2011 (IDEAM 2012; ASTM 2008; Colina et al., 2009).

\section{Solución Numérica del Modelo del Reactor}

La ecuación de balance de materia de la zona de reacción (ec. 4) es una ecuación integro-diferencial en función de la concentración del 4-clorofenol y acoplada al balance de materia en zona oscura (ec. 5), a las ecuaciones hidrodinámicas (ec. 10 a 13), y la LVRPA (ec. 21):

$$
\left.\frac{\partial C_{i}}{\partial Z}\right|_{\tau \text { pass }}=-\frac{2}{v_{z, a v}} \frac{\alpha_{1}}{\kappa_{P}}\left(\frac{1}{v_{T}}\right) \int_{v_{R}}\left[-1+\sqrt{1+\frac{\kappa_{P}}{\alpha_{1}} \Phi_{g} \hat{E}_{g\left(\lambda \rightarrow \lambda^{\prime}\right)}^{a}}\right] \theta_{i}^{L-H} d v
$$

El sistema se solucionó a través del método Runge-Kutta de $4^{\circ}$ orden con un esquema de duplicación diferencial para el cálculo del error local de integración. Los parámetros cinéticos del 4-cloforfenol y el valor del rendimiento cuántico fueron obtenidos de Mueses et al, 2013. El mismo algoritmo fue utilizado para las simulaciones del sistema.

\section{Validación del Modelo}

El modelo matemático y la simulación fueron validados con datos experimentales a diferentes condiciones de operación para la degradación fotocatalítica de 4-clorofenol (Merck® grado reactivo) utilizando $\mathrm{TiO}_{2}-\mathrm{P} 25$ de Degussa, en un reactor solar CPC a escala piloto ubicado en la Plataforma Solar de la Escuela de Ingeniería Química, Universidad del Valle, Cali-Colombia ( $3^{\circ} 30^{\prime}$ latitud norte). Los parámetros de diseño del reactor, condiciones de operación y datos experimentales se reportaron en trabajos previos (Mueses et al., 2013; Suaterna et al., 2012).

\section{RESULTADOS Y DISCUSIÓN}

El desempeño de la velocidad volumétrica de absorción de fotones (VRPA), muestra la existencia de alta concentración de radiación sobre la capa límite del reactor cercano a la pared (lim $r_{P}^{\prime} \rightarrow 0$ ) (véase Figura 2). El espesor óptico de la suspensión favorece los efectos de apantallamiento; para el CPC, valores de $\delta$ cercanos a cero muestran un perfil de alta energía, que disminuyen con el incremento del espesor óptico $\delta$. Esto puede afectar notablemente el rendimiento global del foto-reactor debido a la disminución de la energía disponible para la activación en zonas de espesor óptico altos.

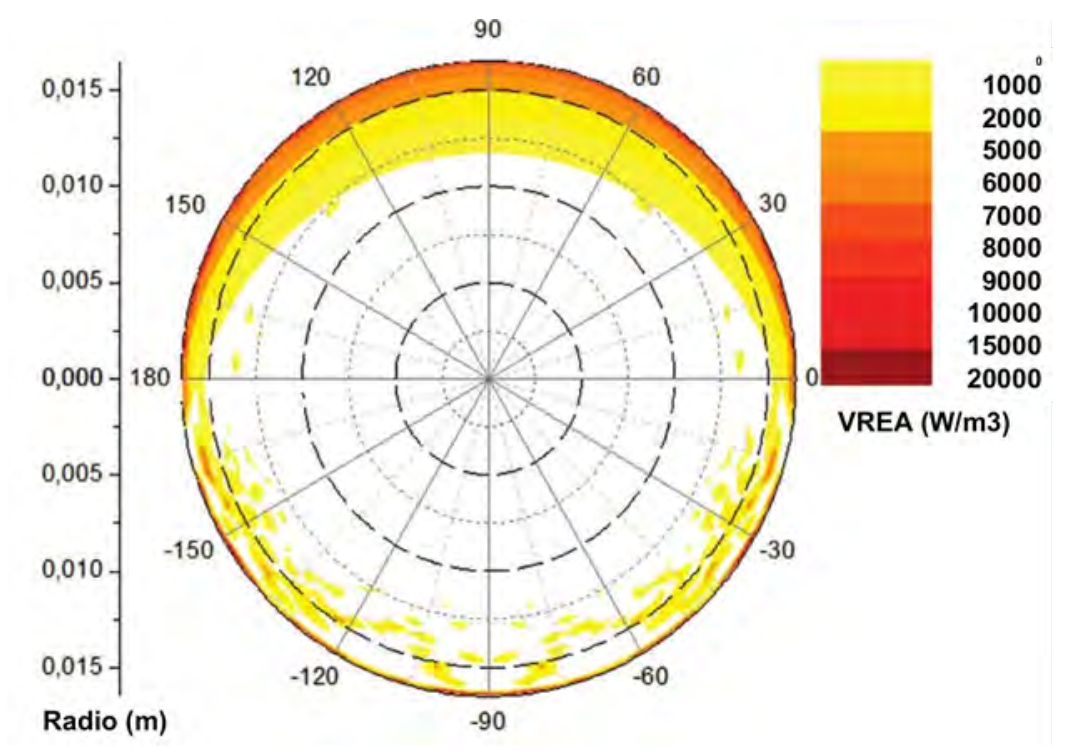

Fig. 2. Simulación de campo radiante un reactor solar CPC. 
Este comportamiento del reactor toma aún mayor importancia para las limitaciones dinámicas del sistema, dado que la hidrodinámica y los procesos de transferencia de masa pueden sobreponerse sobre los efectos del flujo de radiación cerca las paredes del reactor, generando competencia entre las limitaciones de película en la pared y su influencia sobre la conversión del sistema. En la Figura 3 se muestra la simulación del perfil de velocidad para la mitad del tubo de reacción. Es evidente que los esfuerzos cortantes a espesores ópticos cercanos a cero, donde la energía disponible para la activación es alta, implica velocidades cercanas a la pared (nulas) por tanto, los efectos hidrodinámicos afectan radicalmente el desempeño del foto-reactor. Por su parte, zonas de mayor turbulencia y alta velocidad (cercanas al eje de simetría donde los esfuerzos cortantes son nulos) potencialmente representarían condiciones favorables de mezcla perfecta y por tanto incrementos de la conversión global; sin embargo, estas zonas están limitadas por la disponibilidad fotónica para activación.

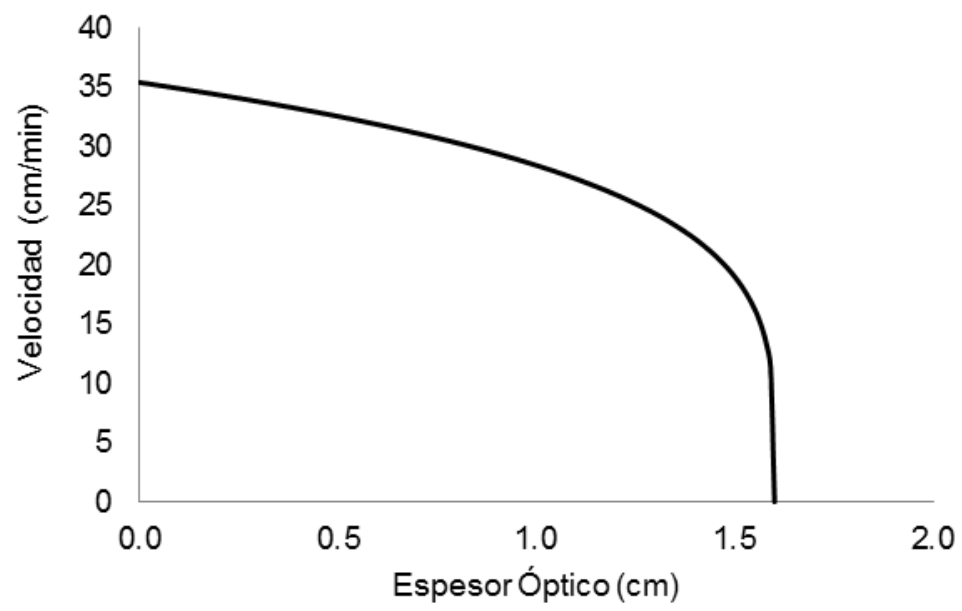

Fig. 3. Simulación del perfil hidrodinámico dentro del tubo para una velocidad media global de $75 \mathrm{gal} / \mathrm{min}$.

\section{Predicciones}

El desempeño experimental y cinético de la degradación fotocatalítica solar heterogénea a escala piloto del 4-cloro fenol fue discutida y analizada adecuadamente en un trabajo previo (Suaterna et al., 2012). En la presente investigación se muestra la capacidad predictiva del modelo global del reactor como acople de los modelos de campo radiante, velocidad de reacción, rendimiento cuántico e hidrodinámico; además de la robustez del algoritmo propuesto para la simulación. En Figura 4 se presenta el desempeño del modelo matemático en la predicción de datos experimentales.

Los errores de discrepancia residual fueron inferiores al 1.5\%. Este resultado es relevante considerando que la simulación del campo de radiación es complejo debido a la alta variabilidad de las condiciones atmosféricas que afectan la energía incidente al sistema reactor (Mueses et al., 2013 y Colina-Márquez, et al., 2009). Se muestra que el modelo cinético implementado permite extender su aplicación a diferentes condiciones de operación logrando resultados altamente eficientes en términos predictivos. La alta efectividad se logra por la variabilidad que presenta el rendimiento cuántico del sistema por corrección con las condiciones de carga de catalizador, $\mathrm{pH}$ y flujo luminoso reflejado en la LVRPA, Ec. 9 (Mueses et al, 2013). La variación de la LVRPA en función de la carga de catalizador se presenta en la Figura 4 d). Para este comportamiento, la LVRPA a bajas concentraciones de catalizador presenta los máximos valores posibles en el sistema CPC, esto debido a que los efectos de scattering favorecen la actividad fotónica y se sobreponen a los efectos de apantallamiento; no obstante, el incremento de la carga de catalizador favorece el apantallamiento y la actividad del catalizador disminuye.

Este resultado se aprecia claramente en la simulación de la degradación del 4-clorofenol (figuras 4 a, b, c) donde para concentraciones altas de catalizador se disminuye la degradación. Para concentraciones muy bajas de catalizador, la actividad catalítica se ve afectada por déficit de electrones-huecos fotogenerados, por tanto se presenta déficit de radicales hidroxilo y de este modo se disminuye también el desempeño de la fotodegradación. Desde el punto de vista óptico concentraciones alrededor de $0.35 \mathrm{~g} / \mathrm{L}$ son más adecuadas para este tipo de reactores y procesos (Colina-Márquez, et al., 2010); sin embargo, los efectos cinéticos, la limitación de la transferencia de masa y los efectos hidrodinámicos generan discrepancias con estos resultados (Mueses et al., 2013). 

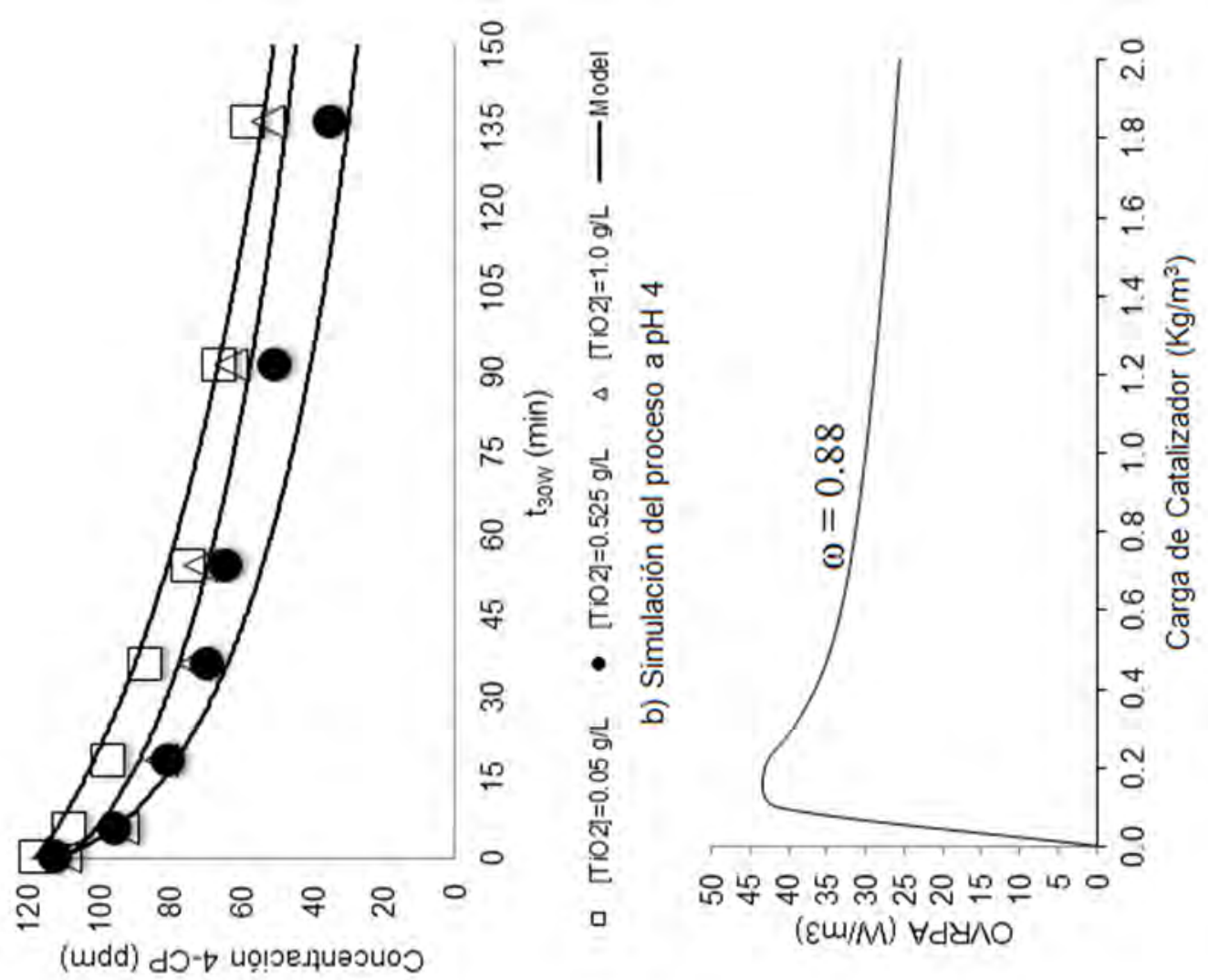

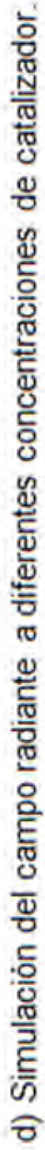
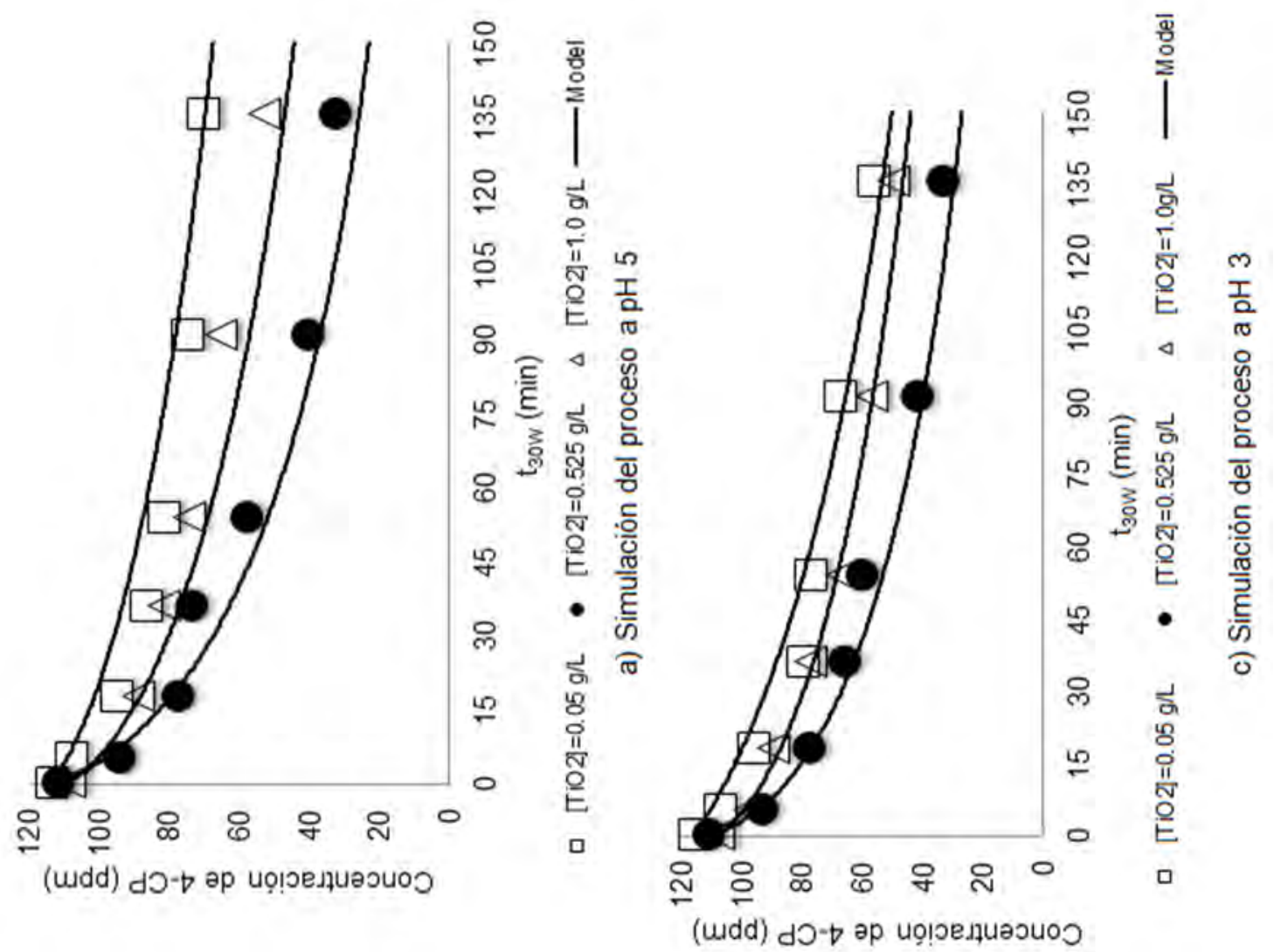

Fig. 4. Simulación de la fotodegradación de 4-clorofenol a diferentes condiciones de operación de $\mathrm{pH}$ y carga de catalizador. 
Finalmente, la estructura matemática del modelo (ec. 5 a 7) como función de un modelo modificado del tipo Langmuir-Hinshelwood, pero con corrección por efectos de flujo luminoso, adsorción molecular y rendimiento cuántico, garantiza la efectividad del modelo en la predicción de datos experimentales, incluso a concentraciones altas de substrato (120 ppm como el caso de este estudio), una limitante fundamental en modelos del tipo Langmuir que se utilizan comúnmente a escala solar en tratamiento de aguas contaminadas con este tipo de compuestos (Colina-Márquez, et al. 2009).

Los resultados encontrados para la degradación fotocatalítica del 4-clorofenol a escala solar en un reactor CPC garantizan la efectividad en la extensión del modelo cinético aplicado. La naturaleza global de los parámetros del modelo, permitieron cuantificar de manera precisa el comportamiento del proceso incluso con variaciones de la radiación incidente debido a efectos atmosféricos. Este enfoque de modelado, puede ser implementado en la evaluación del tratamiento de aguas residuales industriales no caracterizadas a diferentes condiciones de operación, y en la evaluación de los reactores solares, lo cual es una contribución fundamental en ingeniería de diseño, en el montaje y operación de reactores fotocatalíticos a gran escala.

\section{CONCLUSIONES}

El modelado matemático y la simulación de la degradación fotocatalítica heterogénea con $\mathrm{TiO}_{2}-\mathrm{P} 25$ de 4clorofenol a escala piloto en un reactor CPC, fue formulada por acoplamiento de un modelo de campo de absorción-dispersión (SFM), un modelo cinético basado en ataque de radicales hidroxilo con estructura matemática del tipo Langmuir-Hinshelwood modificado, un modelo de rendimiento cuántico y el modelo del reactor. Este acoplamiento garantizó alta efectividad predictiva a diferentes condiciones de operación con errores inferiores a 1.5\% en comparación con datos experimentales utilizados para la validación. El modelo es capaz de describir la fotodegradación del componente incluso a composiciones altas del substrato, una limitante fundamental en modelos similares basados en Langmuir-Hinshelwood.

\section{AGRADECIMIENTOS}

Los autores agradecen a la Universidad de Cartagena y a la Universidad del Valle por la financiación de esta publicación; igualmente agradecen a Colciencias por la financiación de estudios doctorales.

\section{REFERENCIAS}

Cassano A. y Alfano, O., Reaction engineering of suspended solid heterogeneous photocatalytic reactors, Catalysis Today: 58, 167-197 (2000).

Colina-Márquez, J., F. Machuca-Martínez y G. Li Puma, Photocatalytic mineralization of commercial herbicides in a pilot-scale solar CPC reactor: Photoreactor modeling and reaction kinetics constants independent of radiation field, Environmental Science and Technology: 43, 8953-8960 (2009).

Colina-Márquez, J., F. Machuca-Martínez y G. Li Puma, Radiation absorption and optimization of solar photocatalytic reactors for environmental applications, Environmental Science and Technology: 44, 51125120 (2010).

IDEAM, Atlas De Radiación Solar De Colombia-2005, Ministerio del Medio Ambiente: Bogotá (Colombia), 28-40, available on-line https://documentacion.ideam.gov.co/openbiblio/Bvirtual/019649/019649.htm

Li Puma, G. y A. Brucato, Dimensionless analysis of slurry photocatalytic reactors using a two-flux and sixflux radiation absorption-scattering models, Catalysis Today 122, 78-90 (2007).

Machuca-Martínez, F., J. Colina-Márquez y M. Mueses, Determination of quantum yield in a heterogeneous photocatalytic system using a fitting-parameters model, Journal of Advanced Oxidation Technologies: 11 (1), $42-48$ (2008).

Malato, S., J. Blanco, A. Vidal, P. Fernández, y A. Agüera, Solar photocatalytic mineralization of commercial pesticides: acrinathrin, Chemosphere 40, 403-409 (2000).

Malato, S., J. Blanco, C. Richter, B. Milow y M. Maldonado, Preindustrial experience in solar photocatalytic mineralization of real wastewaters: Application to pesticide container recycling, Water Science and Technology: 40,123-130 (1999). 
Mueses, M., F. Machuca y G. Li Puma, Effective Quantum Yield and Reaction Rate Model for Evaluation of Photocatalytic Degradation of Water Contaminants in Heterogeneous Pilot-Scale Solar Photoreactors, Chemical Engineering Journal: 215-216, 937-947 (2013).

Mueses, M. y F. Machuca-Martínez, A new concept of quantum yield for photocatalytic heterogeneous reactions kinetics, In $16^{\text {th }}$ International Conference on $\mathrm{TiO}_{2}$ Photocatalysis: Fundamentals and Applications, San Diego-CA, USA, Nov 07-10 (2011).

Satuf, M., R. Brandi, A. Cassano y O. Alfano, Photocatalytic degradation of 4-chlorophenol: A kinetic study, Applied Catalysis B: 82, 37-49 (2008).

Standard Tables for Reference Solar Spectral Irradiance, ASTM G173 03 (2008); ASTM International: West Conshohocken, PA, 2003. EDT 2012-Reapproved (2008).

Suaterna, N., C. Insignares, R. Camargo y M. Mueses, Solar photocatalytic degradation of 4-chloropenol a pilot-scale, Información Tecnológica: 23 (6),13-24 (2012).

Zalazar, C., R. Romero, C. Martin y A. Cassano, Photocatalytic intrinsic reaction kinetics I: Mineralization of dichloroacetic acid, Chemical Engineering Science: 60, 5240-5254 (2005). 